\title{
AN EXISTENCE THEOREM FOR THE MULTI-FLUID STOKES PROBLEM
}

\author{
BY \\ A. NOURI (Laboratoire J. A. Dieudonné, Université de Nice, Parc Valrose, 06108, Nice Cédex 2, \\ France), \\ F. POUPAUD (Laboratoire J. A. Dieudonné, Université de Nice, Parc Valrose, 06108, Nice Cédex \\ 2, France), \\ AND \\ Y. DEMAY (Institut Non-Linéaire de Nice, Sophia Antipolis, 06560 Valbonne Cédex, France)
}

\begin{abstract}
Time-dependent flows of viscous incompressible immiscible fluids are studied in the limit of vanishing Reynolds numbers. The velocity fields associated to each fluid solve Stokes equations in a time-dependent domain. Classical immiscibility conditions on the varying fluids interfaces are taken into account by a new formulation of the problem: the viscosity solves a transport equation and the velocity field solves a Stokes problem with this nonconstant viscosity. This formulation, based on the use of a pseudoconcentration function, has already been used for numerical computations (see [9] and [4]). For this nonlinear system of equations, existence of solutions is proved, using the Schauder fixed point theorem and the concept of renormalized solutions introduced recently by R. J. DiPerna and P. L. Lions.
\end{abstract}

1. Introduction. Fluids interface computation has received increasing attention in recent years in the domain of polymer processing because of the proliferation of products that are manufactured using various coating and multi-layer extrusion technologies. Coextrusion, i.e., extrusion of several polymers, is important in the polymer industry since it can lead to more economical production of flat film than the conventional lamination. This allows one to obtain products combining properties of different polymers such as low cost, surface aspect, or impermeability (barrier materials). In this case, due to the high viscosity of polymer melts, the Reynolds number vanishes. Other factors such as density differences and tension between interfaces may also play a role but are considered as negligible in polymer processing:

From a numerical point of view, to compute fluid interfaces, at least two strategies are possible: tracking and capturing. The first strategy tracks the interface and requires full or partial remeshing of the domain in order to follow the interface with iteration

Received June, 1994.

1991 Mathematics Subject Classification. Primary 35Q30, 76T05. 
or time. On the other hand, the capturing strategy requires a single mesh and the different interfaces are determined by the pseudoconcentration function computed in the whole domain. This however requires the solution of a supplementary partial differential equation of hyperbolic type. This strategy was adopted in the volume of fluid method (VOF) of Hirt and Nichols ([10]). The reader is referred to the paper of Shen ([9]) for a review of these methods in the context of injection moulding.

From a mathematical point of view, the flow of $N$ fluids occupying time-dependent subdomains $\Omega_{k}(t), k=1, \ldots, N$, of a fixed domain $\Omega \subset R^{D}, D=2$ or 3 , is considered. In each subdomain the fluid behaviour, i.e., the relationship between the strain and stress tensors, incompressibility and equilibrium, neglecting inertia forces, lead to the Stokes equations. At the interfaces, transmission conditions are the continuity of the velocity and the normal component of the stress tensor. These conditions are obtained by variational considerations. The nonmiscibility condition at interfaces is shown to be equivalent to a transport equation on the whole domain for the viscosity, or of other rheological constants in the case of more complex rheology. The aim of this paper is to derive existence of weak solutions for the nonlinear system of equations obtained by coupling this transport equation with Stokes equations. Unfortunately, smoothness properties of the velocity field do not allow one to obtain a classical solution for the transport equation. Therefore, using the concept of renormalized solutions introduced by R. J. DiPerna and P. L. Lions is necessary (see [3]). New difficulties arise when dealing with boundary conditions. They are overcome by constructing "extended" solutions. It allows the precise control of the incoming and outgoing fluxes. Then the existence proof of solutions for the coupled system is obtained by means of the Schauder fixed point theorem. The compactness property of the map, whose fixed points are solutions of the multi-fluid problem, is proved thanks to a compensated compactness lemma. We point out that this technique applies for the full incompressible Navier-Stokes equations. A further paper will present results in this direction.

In Sec. 2, we derive the weak formulation we use, with classical interface conditions and present the main result. Section 3 is devoted to the Stokes problem with a given nonconstant viscosity. We prove a uniqueness and existence result for this linear problem, with standard techniques. Section 4 deals with the transport problem when the velocity fields are not assumed to be Lipschitz. We prove existence and uniqueness of extended solutions by means of techniques developed in [3]. Finally, Sec. 5 concludes the paper by giving a proof of the main theorem. This proof is based on results derived in the previous sections and on compensated compactness techniques.

2. The multi-fluid Stokes problem. Let us consider $N$ viscous fluids with viscosities $\eta_{k}\left(1 \leq k \leq N, \eta_{1} \leq \eta_{2} \leq \cdots \leq \eta_{N}\right)$ flowing in an open set $\Omega$ of $R^{D}$. The $k$ th fluid occupies at time $t$ the open subdomain $\Omega_{k}(t)$. Denote by $\mathbf{u}_{k}(t, \mathbf{x})$ the velocity vector $\left(\mathbf{x} \in \Omega_{k}(t)\right)$. The velocity is globally defined on $\Omega$ by

$$
\mathbf{u}=\mathbf{u}_{k}(t, \mathbf{x}) \quad \text { for } \mathbf{x} \in \Omega_{k}(t), k=1, \ldots, N
$$


In the following, letters without subscripts, such as $\gamma$, denote functions defined on the whole domain $\Omega$, and the same letters with subscripts, such as $\gamma_{k}$, denote the restriction of these functions to the subdomains $\Omega_{k}(t), k=1, \ldots, N$.

2.1. Stokes equations. The strain tensor $\varepsilon$ is defined by

$$
\varepsilon\left(\mathbf{u}_{k}\right)=\frac{1}{2}\left(\nabla \mathbf{u}_{k}+\nabla \mathbf{u}_{k}^{t}\right), \quad \mathbf{x} \in \Omega_{k}(t) .
$$

The fluid incompressibility is expressed by

$$
\operatorname{div}\left(\mathbf{u}_{k}\right)=0 \text { or } \operatorname{Tr}\left(\varepsilon\left(\mathbf{u}_{k}\right)\right)=0
$$

The Newtonian behaviour gives the constitutive equation for the stress tensor $\boldsymbol{\sigma}_{k}$ :

$$
\boldsymbol{\sigma}_{k}=2 \eta_{k} \varepsilon\left(\mathbf{u}_{k}\right)-p_{k} \mathbf{I d}
$$

Inertia being neglected and gravity not considered, the equilibrium equation is

$$
\operatorname{div}\left(\boldsymbol{\sigma}_{k}\right)=0
$$

Stokes equations are classically obtained by substituting Eqs. (2.1) and (2.2) in the previous equation and using that $\mathbf{u}_{k}$ is divergence free:

$$
\eta_{k} \Delta \mathbf{u}_{k}-\nabla p_{k}=0, \quad \operatorname{div}\left(\mathbf{u}_{k}\right)=0
$$

2.2. Transmission conditions. Denote by $\mathbf{h}_{m}(t)$ an interface between fluids $k$ and $l$. The classical transmission conditions are the continuity of the velocity $\mathbf{u}$ and of the normal component of the stress tensor at the interface:

$$
\begin{aligned}
\mathbf{u}_{k}(t, \mathbf{x}) & =\mathbf{u}_{l}(t, \mathbf{x}), & & \mathbf{x} \in \mathbf{h}_{m}(t), \\
\boldsymbol{\sigma}_{k}(t, \mathbf{x}) \cdot \mathbf{n} & =\boldsymbol{\sigma}_{l}(t, \mathbf{x}) \cdot \mathbf{n}, & & \mathbf{x} \in \mathbf{h}_{m}(t) .
\end{aligned}
$$

The vector $\mathbf{n}$ is the normal in $\mathbf{h}_{m}$ at point $\mathbf{x}$ and time $t$. These conditions correspond to those obtained in multi-material elasticity (see [1]). Let us remark that if (2.5) is interpreted for almost every $t$ as a trace equality in $H^{1 / 2}\left(\mathbf{h}_{m}(t)\right)$ and if the functions $\mathbf{u}_{k}$ belong to $H^{1}\left(\Omega_{k}(t)\right)$, then $\mathbf{u}$ belongs to $H^{1}(\Omega)$. In the same way, the continuity of the stress normal component (2.6), interpreted for almost every $t$ as an equality in $H^{-1 / 2}\left(\mathbf{h}_{m}(t)\right)$, implies that the "globally" defined stress tensor $\boldsymbol{\sigma}$ belongs to $H=\{\boldsymbol{\sigma} \in$ $\left(L^{2}(\Omega)\right)^{6} ; \operatorname{div}(\boldsymbol{\sigma})=0$ in $\left.D^{\prime}(\Omega)\right\}$. These remarks will allow us to derive a weak formulation of the multi-fluid problem. Indeed, we have

LEMMA 2.1. Let $\Omega_{m}$ be Lipschitz bounded subsets of a bounded set $\Omega=\bigcup_{m=1}^{N} \Omega_{m}$, and let $\eta$ be the function defined by $\eta=\eta_{m}$ on $\Omega_{m}$. Then for every $p$ in $L^{2}(\Omega)$ and $u$ in $H^{1}(\Omega)$, the following propositions are equivalent:

(i) $\boldsymbol{\sigma}=\eta \varepsilon(u)-p \mathrm{Id}, \operatorname{div}(\boldsymbol{\sigma})=0, \operatorname{div}(u)=0$.

(ii) $\eta_{k} \Delta u_{k}-\nabla p_{k}=0$ in $\Omega_{k}, \operatorname{div}\left(u_{k}\right)=0$ in $\Omega_{k}$, $u_{k / \mathbf{h}_{m}}=u_{l / \mathbf{h}_{m}}$ in $H^{1 / 2}\left(\mathbf{h}_{m}\right)$, $\boldsymbol{\sigma}_{k} \cdot \mathbf{n}_{/ \mathbf{h}_{m}}=\boldsymbol{\sigma}_{l} \cdot \mathbf{n}_{/ \mathbf{h}_{m}}$ in $H^{-1 / 2}\left(\mathbf{h}_{m}\right)$. 
2.3. The immiscibility condition. Define

$$
\begin{gathered}
\Pi=[0, T] \times \Omega ; \quad \Pi_{k}=\left\{(t, \mathbf{x}) \text { such that } 0 \leq t \leq T \text { and } \mathbf{x} \in \Omega_{k}(t)\right\}, \\
\mathbf{H}_{m}=\left\{(t, \mathbf{x}) \text { such that } 0 \leq t \leq T \text { and } \mathbf{x} \in \mathbf{h}_{m}(t)\right\} .
\end{gathered}
$$

Denote by $\mathbf{N}$ the normal to $\Pi_{k}$ at the boundary and $\mathbf{U}=(1, \mathbf{u}(t, \mathbf{x}))$. For the timedependent case, the immiscibility condition is classically written (see [1]):

$$
\mathbf{U} \cdot \mathbf{N}=0, \quad \text { for }(t, \mathbf{x}) \in \mathbf{H}_{m} .
$$

The interface $\mathbf{H}_{m}$ is locally described as the graph of a Lipschitz function $h$ :

$$
x_{d}=h\left(t, \mathbf{x}^{\prime}\right) \quad \text { with } \mathbf{x}=\left(x_{d}, \mathbf{x}^{\prime}\right)
$$

and

$$
\mathbf{u}=\left(u_{d}, \mathbf{u}^{\prime}\right), \quad \mathbf{x}^{\prime} \in R^{D-1}, \quad \mathbf{u}^{\prime}(\mathbf{x}) \in R^{D-1} .
$$

The previous equation becomes

$$
\frac{\partial h}{\partial t}-u_{d}+\mathbf{u}^{\prime} \cdot \nabla_{\mathbf{x}^{\prime}} h=0
$$

Let us define the viscosity function as

$$
\eta(t, \mathbf{x})=\eta_{k} \quad \text { if } \mathbf{x} \in \Omega_{k}(t) .
$$

These conditions at the interfaces can be expressed as a weak formulation of a transport equation of the viscosity on the whole domain:

$$
\frac{\partial \eta}{\partial t}+\mathbf{u} \cdot \nabla \eta=0, \quad(t, \mathbf{x}) \in \Pi
$$

In order to give a precise mathematical meaning to these statements, we first derive a trace property.

LEMMA 2.2. Let $\Pi_{k}$ be a Lipschitz bounded set of $\Pi$ :

$$
\Pi_{k}=\left\{(t, \mathbf{x}) \in R^{D+1}, t \in(0, T), \mathbf{x} \in \Omega_{k}(t)\right\} .
$$

Then there is a continuous trace mapping $\Gamma_{k}$ from $V_{1}\left(\Pi_{k}\right)$ onto $V_{1 / 2}\left(\partial \Pi_{k}\right)$, where $V_{1}\left(\Pi_{k}\right)$ and $V_{1 / 2}\left(\partial \Pi_{k}\right)$ are defined in the following way:

$$
\begin{aligned}
V_{1}\left(\Pi_{k}\right) & =\left\{\mathbf{u}_{/ \Pi_{k}} ; \mathbf{u} \in L^{\infty}\left(0, T ;\left(H^{1}(\Omega)\right)^{D}\right)\right\}, \\
V_{1 / 2}\left(\partial \Pi_{k}\right) & =\left\{\mathbf{v} \circ \boldsymbol{\psi} ; \mathbf{v} \in L^{\infty}\left(0, T ;\left(H^{1 / 2}\left(\partial B_{1}\right)\right)^{D}\right)\right\},
\end{aligned}
$$

with

$$
B_{1}=\left\{\mathbf{x} \in R^{D} ;|\mathbf{x}|<1\right\}, \quad \boldsymbol{\psi} \in \operatorname{Lip}\left([0, T] \times \bar{B}_{1} ; \bar{\Pi}_{k}\right), \quad \boldsymbol{\psi} \text { is one-to-one and onto. }
$$

Proof. Denote by $\gamma$ the trace mapping from $\left(H^{1}\left(B_{1}\right)\right)^{D}$ into $\left(H^{1 / 2}\left(\partial B_{1}\right)\right)^{D} . \Gamma_{k}$ is defined by

$$
\Gamma_{k}\left(\mathbf{u}_{k}\right)=\gamma\left(\mathbf{u}_{k} \circ \boldsymbol{\psi}^{-1}\right) \circ \boldsymbol{\psi}, \quad \mathbf{u}_{k} \in V_{1}\left(\Pi_{k}\right) .
$$

The equivalence between (2.7) and (2.10) is expressed in

Lemma 2.3. Assume that $\Pi_{k}, k=1, \ldots, N$, are Lipschitz domains and that $\mathbf{u}$ belongs to $L^{\infty}\left(0, T ;\left(H^{1}(\Omega)\right)^{D}\right)$. Then the following conditions are equivalent:

(i) $\Gamma_{k}\left(\mathbf{u}_{k}\right) \cdot \mathbf{N}=0=\Gamma_{l}\left(\mathbf{u}_{1}\right) \cdot \mathbf{N}$ on $\mathbf{H}_{m}$; 
(ii) $\frac{\partial \eta}{\partial t}+\mathbf{u} \cdot \nabla \eta=0$ in $D^{\prime}(\Pi)$.

Proof. In a weak sense Eq. (2.10) means

$$
\int_{\Pi} \eta\left(\frac{\partial \varphi}{\partial t}+\mathbf{u} \cdot \nabla \varphi\right) d t d x=\int_{\Pi} \eta\left(\mathbf{U} \cdot \nabla_{t, \mathbf{x}} \varphi\right) d t d x=0 \quad \text { for every } \varphi \text { in } C_{0}^{\infty}(\Pi) \text {. }
$$

If, for example, $N=2$ and $\Pi=\Pi_{1} \cup \Pi_{2} \cup \mathbf{H}$ and if the interface $\mathbf{H}$ is smooth, (2.11) becomes, since $\varphi$ vanishes on the boundary of $\Pi$ :

$$
\int_{\Pi_{1}} \eta\left(\frac{\partial \varphi}{\partial t}+\mathbf{u} \cdot \nabla \varphi\right) d t d x+\int_{\Pi_{2}} \eta\left(\frac{\partial \varphi}{\partial t}+\mathbf{u} \cdot \nabla \varphi\right) d t d x=0
$$

Using the divergence theorem and Lemma 2.2, we obtain

$$
\left(\eta_{1}-\eta_{2}\right) \int_{H} \mathbf{U} \cdot \mathbf{N} \varphi d t d x=0 \quad \text { for every } \varphi \text { in } C_{0}^{\infty}(\Pi),
$$

which concludes the proof.

Hence, the multi-fluid Stokes problem can be written as a Stokes problem with a variable viscosity, coupled with a transport equation for the viscosity. Indeed, combining Lemma 2.1 and Lemma 2.2 we obtain:

Proposition 2.1. Assume that the subdomains $\Pi_{k}=\left\{(t, \mathbf{x}) \in \Pi ; \eta(t, \mathbf{x})=\eta_{k}\right\}$ are Lipschitz. Then the following properties are equivalent:

(i) $\mathbf{u}_{k} \in V_{1}\left(\Pi_{k}\right), p_{k} \in V_{0}\left(\Pi_{k}\right)$,

$$
\begin{aligned}
& \eta_{k} \Delta \mathbf{u}_{k}-\nabla p_{k}=0 \text { in } \Pi_{k}, \operatorname{div}\left(\mathbf{u}_{k}\right)=0 \text { in } \Pi_{k}, \\
& \Gamma_{k}\left(\mathbf{u}_{k}\right)=\Gamma_{l}\left(\mathbf{u}_{l}\right) \text { on } \mathbf{H}_{m}, \\
& \Gamma_{k}\left(\mathbf{U}_{k}\right) \cdot \mathbf{N}=\Gamma_{l}\left(\mathbf{U}_{l}\right) \cdot \mathbf{N}=0 \text { on } \mathbf{H}_{m}, \\
& \Sigma_{k / \partial \Pi_{k}} \cdot \mathbf{N}=0=\Sigma_{l / \partial \Pi_{l}} \cdot \mathbf{N} \text { on } \mathbf{H}_{m},
\end{aligned}
$$

where $\mathbf{U}={ }^{t}(1, \mathbf{u}), \Sigma={ }^{t}(1, \sigma), \sigma=\eta \varepsilon(\mathbf{u})-p$ Id, and $\Sigma_{k / \partial \Pi_{k}}$ denotes the trace of $\Sigma$ in $H^{-1 / 2}\left(\partial \pi_{k}\right)$.

(ii) $\mathbf{u} \in L^{\infty}\left(0, T ; H^{1}(\Omega)\right), p \in L^{\infty}\left(0, T ; L^{2}(\Omega)\right)$,

$\operatorname{div}(\eta \varepsilon(\mathbf{u})-p \mathrm{Id})=0, \operatorname{div}(\mathbf{u})=0$,

$\frac{\partial \eta}{\partial t}+\mathbf{u} \cdot \nabla \eta=0$ in $\Pi$.

We shall use (ii) as a weak formulation of the multi-fluid problem.

REMARK. The strict equivalence between (i) and (ii) is only obtained when the interfaces are assumed to be smooth. But in the following we will construct a weak solution of (ii). Especially, the viscosity $\eta$ only belongs to $L^{\infty}(\Pi)$. Therefore, the subdomains $\Pi_{k}=\eta^{-1}\left(\eta_{k}\right)$ have no reason to be smooth. Fractal interfaces may occur. Then interfacial transmission conditions (i) would no longer have any meaning. However, even in that situation, weak solutions of (ii) are mechanically admissible. Indeed, the rheological constant $\eta$ is always transported by the fluid as it is expressed by the transport equation and through every smooth surface there is continuity of velocities and of the normal component of the stress tensor, in the sense of traces in $H^{1 / 2}$ and $H^{-1 / 2}$ respectively.

We now state the main result of this paper: 
THEOREM 2.1. Let $\Omega$ be a bounded Lipschitz open set of $R^{D}$, whose boundary $\partial \Omega$ splits into

$$
\partial \Omega=\Gamma_{\text {in }} \cup \Gamma_{0} \cup \Gamma_{\text {out }},
$$

where $\Gamma_{\text {in }}$ and $\Gamma_{\text {out }}$ are separated from each other. That means that there exist two open sets $\Omega_{\text {in }}$ and $\Omega_{\text {out }}$ such that

$$
\Gamma_{\text {in }} \subset \Omega_{\text {in }}, \quad \Gamma_{\text {out }} \subset \Omega_{\text {out }}, \quad \Omega_{\text {in }} \cap \Omega_{\text {out }}=\varnothing \text {. }
$$

Let $\mathbf{u}_{0} \in L^{2}((0, \infty) \times \partial \Omega)$ be such that there exists $\mathbf{U}_{0} \in L^{\infty}\left(0, \infty ; C_{0}^{1}\left(R^{D}\right)\right)$ verifying

$$
\begin{gathered}
\mathbf{U}_{0}=\mathbf{u}_{0} \text { on }(0, \infty) \times \partial \Omega, \quad \operatorname{div}\left(\mathbf{U}_{0}\right)=0, \\
\mathbf{U}_{0} \cdot \mathbf{n}<0, \quad t \geq 0, \mathbf{x} \in \Gamma_{\mathrm{in}}, \\
\mathbf{U}_{0} \cdot \mathbf{n}=0, \quad t \geq 0, \mathbf{x} \in \Gamma_{0}, \\
\mathbf{U}_{0} \cdot \mathbf{n}>0, \quad t \geq 0, \quad \mathbf{x} \in \Gamma_{\text {out }},
\end{gathered}
$$

where $\mathbf{n}$ denotes the outward normal on $\partial \Omega$. Let $0<\eta_{1}<\cdots<\eta_{n}$, and $\eta_{0}$ and $\eta_{\text {in }}$ belonging to $L^{\infty}(\Omega)$ and $L^{\infty}\left((0, \infty) \times \Gamma_{\text {in }}\right)$ respectively, be such that

$$
\left(\eta_{0}, \eta_{\text {in }}\right) \in\left\{\eta_{1}, \ldots, \eta_{n}\right\} \text { a.e. }
$$

Then there is at least one solution $(\mathbf{u}, \eta, p)$ of the coupled system of equations:

$$
\begin{aligned}
\operatorname{div}\left(\eta(t, \mathbf{x})\left(\nabla \mathbf{u}+\nabla \mathbf{u}^{t}\right)\right) & =\nabla p, & & t>0, \mathbf{x} \in \Omega, \\
\operatorname{div}(\mathbf{u}) & =0, & & t>0, \mathbf{x} \in \Omega, \\
\mathbf{u} & =\mathbf{u}_{0}, & & t>0, \mathbf{x} \in \partial \Omega,
\end{aligned}
$$

and

$$
\begin{array}{rlrl}
\frac{\partial \eta}{\partial t}+\mathbf{u} \cdot \nabla \eta & =0, & t>0, \mathbf{x} \in \Omega \\
\eta=\eta_{0}, & t=0, \mathbf{x} \in \Omega, \\
\eta=\eta_{\mathrm{in}}, & t>0, \mathbf{x} \in \Gamma_{\mathrm{in}},
\end{array}
$$

that satisfy

$$
\begin{gathered}
\mathbf{u} \in L^{\infty}\left(0, \infty ;\left(H^{1}(\Omega)\right)^{D}\right) \\
\eta \in L^{\infty}((0, \infty) \times \Omega), \quad \eta \in\left\{\eta_{1}, \ldots, \eta_{n}\right\} \text { a.e. } \\
p \in L^{2}\left(0, \infty ; L^{2}(\Omega)\right) .
\end{gathered}
$$

Before proving this theorem, let us study separately the two problems (2.16) and (2.17). This is the object of the following sections. 
3. The Stokes problem with a given nonconstant viscosity. In this section we assume that $\Omega$ is a Lipschitz bounded set of $R^{D}$. We are concerned with the following Stokes problem:

$$
\begin{aligned}
& \text { Find } \mathbf{u} \in\left(H^{1}(\Omega)\right)^{D}, p \in L_{0}^{2}(\Omega), \text { such that } \\
& \gamma(\mathbf{u})=\mathbf{u}_{0}, \\
& \operatorname{div}(\mathbf{u})=0, \quad \mathbf{x} \in \Omega, \\
& \operatorname{div}\left(\eta(\mathbf{x})\left(\nabla \mathbf{u}+\nabla \mathbf{u}^{t}\right)\right)=\nabla p, \quad \text { in }\left(H^{-1}(\Omega)\right)^{D} .
\end{aligned}
$$

The space $L_{0}^{2}(\Omega)$ is defined by

$$
L_{0}^{2}(\Omega)=\left\{p \in L^{2}(\Omega) \text { such that } \int_{\Omega} p d x=0\right\}
$$

The operator $\gamma$ denotes the trace mapping from $H^{1}(\Omega)$ onto $H^{1 / 2}(\partial \Omega)$. We assume that $\mathbf{u}_{0}$ is the trace of some divergence free function $\mathbf{U}_{0}$ belonging to $\left(H^{1}(\Omega)\right)^{D}$ :

$$
\mathbf{u}_{0}=\gamma\left(\mathbf{U}_{0}\right), \quad \mathbf{U}_{0} \in\left(H^{1}(\Omega)\right)^{D}, \quad \operatorname{div}\left(\mathbf{U}_{0}\right)=0 .
$$

REMARK. If $\Omega$ is smooth, let us say with a $C^{2}$ boundary, then a necessary and sufficient condition in order that there exist a function $\mathbf{U}_{0}$ satisfying (3.2) is

$$
\mathbf{u}_{0} \in H^{1 / 2}(\partial \Omega), \quad \int_{\partial \Omega^{k}} \mathbf{u}_{0} d \sigma(x)=0,
$$

where $\partial \Omega^{k}$ are the connected components of the boundary. Assumption (3.2) is the usual one for obtaining an existence and uniqueness result for the Stokes problem (see [8]).

THEOREM 3.1. Let $\eta$ be a bounded function such that

$$
\eta \in L^{\infty}(\Omega), \quad 0<\eta_{1}<\eta(\mathbf{x})<\eta_{N}<\infty,
$$

and let $\mathbf{u}_{0}$ satisfy (3.2) for some function $\mathbf{U}_{0}$. Then there exists a unique solution $(\mathbf{u}, p)$ of the Stokes problem (3.1). Moreover,

$$
\|\mathbf{u}\|_{H^{1}(\Omega)} \leq 2 C(\Omega) \sqrt{\frac{\eta_{N}}{\eta_{1}}}\left\|\mathbf{u}_{0}\right\|_{H^{1}(\Omega)},
$$

where $C(\Omega)$ is a Poincaré constant.

Proof. We introduce the space

$$
V=\left\{\mathbf{v} \in\left(H_{0}^{1}(\Omega)\right)^{D} ; \operatorname{div}(\mathbf{v})=0\right\},
$$

which is a closed subspace of $\left(H_{0}^{1}(\Omega)\right)^{D}$. Then an equivalent problem to (3.1) is

Find $\mathbf{u}=\mathbf{U}_{0}+\mathbf{v}, \mathbf{v} \in V$, such that:

$$
\int_{\Omega} \eta \varepsilon(\mathbf{v}): \varepsilon(\mathbf{w}) d x+\int_{\Omega} \eta \varepsilon\left(\mathbf{U}_{0}\right): \varepsilon(\mathbf{w}) d x=0 \quad \text { for any } \mathbf{w} \in \mathbf{V} \text {. }
$$

It is easy to check that the bilinear form appearing in (3.6) is coercive, thanks to:

LEMMA (Korn's inequality). Let $\Omega$ be a Lipschitz bounded set. Then for any function $\mathbf{v}$ in $\mathbf{V}$,

$$
\int_{\Omega} \varepsilon(\mathbf{v}): \varepsilon(\mathbf{v}) d x \geq \frac{1}{4}\|\nabla \mathbf{v}\|_{\left(L^{2}(\Omega)\right)^{6}} \geq \frac{C^{2}(\Omega)}{4}\|\mathbf{v}\|_{\left(H^{1}(\Omega)\right)^{d}} .
$$


For a proof of this lemma we refer to [7]. We only point out that we have to assume that $\Omega$ is Lipschitz. Indeed, under such regularity, $\mathbf{V}$ is the closure in $\left(H_{0}^{1}(\Omega)\right)^{D}$ of the space of functions $\mathbf{v}$ of $\left(C_{0}^{\infty}(\Omega)\right)^{D}$ such that $\operatorname{div}(\mathbf{v})=0$ (see [8]), for which (3.7) is easily checked. Hence we obtain existence and uniqueness of a solution of (3.6) that satisfies (3.4). As usual the result for $p$ is obtained by considering

$$
f=\operatorname{div}(\eta \varepsilon(\mathbf{u}))
$$

that is bounded in $H^{-1}(\Omega)$ and satisfies

$$
\langle f, \mathbf{v}\rangle=0 \text { for any } \mathbf{v} \text { in } \mathbf{V} \text {. }
$$

Then there exists a unique $p$ in $L_{0}^{2}(\Omega)$ such that (see [8])

$$
f=\nabla p
$$

4. Transport equations with a non-Lipschitz convective field. Let $\Omega$ be a Lipschitz bounded set of $R^{D}$, whose boundary is split into three connected components $\Gamma_{\text {in }}, \Gamma_{0}$, and $\Gamma_{\text {out }}$. Let us study the following transport problem:

Find $\eta \in L^{\infty}((0, \infty) \times \Omega)$ such that

$$
\begin{array}{ll}
\frac{\partial \eta}{\partial t}+\mathbf{u} \cdot \nabla \eta=0, & t>0, \mathbf{x} \in \Omega, \\
\eta=\eta_{\text {in }}, & t>0, \mathbf{x} \in \Gamma_{\text {in }}, \\
\eta(0, \mathbf{x})=\eta_{0}(\mathbf{x}), & \mathbf{x} \in \Omega .
\end{array}
$$

An existence and uniqueness result for (4.1) is classical when the velocity field $\mathbf{u}$ belongs to $L^{1}(0, T ; \operatorname{Lip}(\Omega))$. It is obtained by the method of characteristics (see [2]). However, in the coupled problem, the natural set for $\mathbf{u}$ is the space $L^{\infty}\left(0, \infty ;\left(H^{1}(\Omega)\right)^{D} \cap N(\operatorname{div})\right)$. In this framework the only available result is due to DiPerna and Lions [3]. They proved the existence and uniqueness result of a weak solution of (4.1) for the Cauchy problem, with $\Omega=R^{D}$, under the weak assumptions $\mathbf{u} \in L^{1}\left(0, T ; W_{\operatorname{loc}}^{1,1}(\Omega)\right), \operatorname{div}(\mathbf{u}) \in L^{1}\left(0, T ; L^{\infty}(\Omega)\right)$. In order to do that, they introduced the new concept of renormalized solutions. Here we have to extend their result to boundary value problems. First we introduce a class of admissible velocity fields:

$$
\mathbf{U}=\left\{\mathbf{u} \in L^{\infty}\left(0, T ;\left(H^{1}(\Omega)\right)^{D}\right) \text { such that } \operatorname{div}(\mathbf{u})=0, \gamma(\mathbf{u})=\gamma\left(\mathbf{U}_{0}\right)\right\}
$$

where $\mathbf{U}_{0} \in L^{\infty}\left(0, T ;\left(C_{0}^{1}\left(R^{D}\right)\right)^{D}\right)$ and satisfies

$$
\begin{aligned}
\operatorname{div}\left(\mathbf{U}_{0}\right)=0, & \\
\mathbf{U}_{0} \cdot \mathbf{n}<0, & \mathbf{x} \in \Gamma_{\text {in }}, \\
\mathbf{U}_{0} \cdot \mathbf{n}=0, & \mathbf{x} \in \Gamma_{0} \\
\mathbf{U}_{0} \cdot \mathbf{n}>0, & \mathbf{x} \in \Gamma_{\text {out }}
\end{aligned}
$$

where $\mathbf{n}$ denotes the outward normal. Then we introduce the space of test functions:

$$
T=\left\{\varphi \in C_{0}^{\infty}\left(R^{D+1}\right) ; \varphi(t, \mathbf{x})=0, t>0, \mathbf{x} \in \Gamma_{\text {out }}\right\},
$$

and the following definitions: 
Definition 4.1. Let $\mathbf{u}$ be in $\mathbf{U}$. Then a weak solution of (4.1) is a function $\eta \in$ $L^{\infty}((0, \infty) \times \Omega)$ such that

$$
\begin{gathered}
\int_{0}^{\infty} \int_{\Omega} \eta\left(\frac{\partial \varphi}{\partial t}+\mathbf{u} \cdot \nabla \varphi\right) d t d x=\int_{\Omega} \eta_{0}(\mathbf{x}) \varphi(0, \mathbf{x}) d x \\
\quad+\int_{0}^{\infty} \int_{\Gamma_{\mathrm{in}}} \eta_{\mathrm{in}}(t, \mathbf{x}) \varphi(t, \mathbf{x})\left|\mathbf{u}_{0} \cdot \mathbf{n}\right| d \sigma(x) d t
\end{gathered}
$$

for every $\varphi$ in $T$.

Definition 4.2. Let $\mathbf{u}$ be in $\mathbf{U}$. Then a renormalized solution of (4.1) is a function $\eta \in L^{\infty}((0, \infty) \times \Omega)$ such that, for any $\beta$ in $C^{1}(R), \beta(\eta)$ is a weak solution of (4.1) with the Cauchy data $\beta\left(\eta_{0}\right)$ and the boundary data $\beta\left(\eta_{\text {in }}\right)$.

In these definitions, we have extensively used the fact that $\mathbf{u}$ is divergence free. Then it is easy to verify that, if $\mathbf{u}$ and $\eta$ are smooth, $\eta$ is a renormalized solution of (4.1). These definitions are not sufficient to control the outgoing flux of the solutions. Therefore, we introduce an auxiliary problem. We first assume that $\Gamma_{\text {in }}$ and $\Gamma_{\text {out }}$ are separated from each other: there exist two open sets $\Omega_{\text {in }}$ and $\Omega_{\text {out }}$ such that

$$
\bar{\Gamma}_{\text {in }} \subset \Omega_{\text {in }}, \quad \bar{\Gamma}_{\text {out }} \subset \Omega_{\text {out }}, \quad \bar{\Omega}_{\text {in }} \cap \bar{\Omega}_{\text {out }}=\varnothing .
$$

Then we extend the velocity field $\mathbf{u}$ in the following way. Let $\theta_{\text {in }}$ and $\theta_{\text {out }}$ be two functions of $C_{0}^{\infty}\left(R^{D+1}\right)$ that satisfy, for some open sets $\omega_{\text {in }}$ and $\omega_{\text {out }}$ such that $\bar{\Gamma}_{\text {in }} \subset \omega_{\text {in }}$ and $\bar{\Gamma}_{\text {out }} \subset \omega_{\text {out }}$,

$$
\begin{aligned}
\theta_{\text {in }}=1 & \text { for } \mathbf{x} \in \omega_{\text {in }}, & \theta_{\text {in }}=0 & \text { for } \mathbf{x} \notin \Omega_{\text {in }}, \\
\theta_{\text {out }}=1 & \text { for } \mathbf{x} \in \omega_{\text {out }}, & \theta_{\text {out }}=0 & \text { for } \mathbf{x} \notin \Omega_{\text {out }} .
\end{aligned}
$$

Define $\tilde{u}$ by

$$
\begin{array}{ll}
\tilde{\mathbf{u}}=\mathbf{u}, & \mathbf{x} \in \Omega, \\
\tilde{\mathbf{u}}=\mathbf{U}_{0}\left(\theta_{\text {in }}+\theta_{\text {out }}\right), & \mathbf{x} \notin \Omega .
\end{array}
$$

Then

LEMma 4.1. The velocity field $\tilde{\mathbf{u}}$ satisfies

$$
\begin{gathered}
\tilde{\mathbf{u}} \in L^{\infty}\left(0, T ; H^{1}\left(R^{D}\right)\right), \quad \operatorname{div}(\tilde{\mathbf{u}}) \in L^{\infty}((0, T) \times \Omega), \\
\tilde{\mathbf{u}}=0, \quad \mathbf{x} \notin \Omega \cup \Omega_{\text {in }} \cup \Omega_{\text {out }} .
\end{gathered}
$$

DEFINITION 4.3. An extended solution of (4.1) is a weak solution of

$$
\begin{array}{ll}
\text { Find } \eta \in L^{\infty}\left((0, \infty) \times\left(\Omega \cup \Omega_{\text {out }}\right)\right) \text { such that } \\
\frac{\partial \eta}{\partial t}+\tilde{\mathbf{u}} \cdot \nabla \eta=0, & t>0, \mathbf{x} \in \Omega \cup \Omega_{\text {out }}, \\
\eta(t, \mathbf{x})=\eta_{\text {in }}(t, \mathbf{x}), & t>0, \mathbf{x} \in \Gamma_{\text {in }}, \\
\eta(0, \mathbf{x})=\tilde{\eta}_{0}(\mathbf{x}), & \mathbf{x} \in \Omega \cup \Omega_{\text {out }},
\end{array}
$$

with $\tilde{\eta}_{0}(\mathbf{x})=\eta_{0}(\mathbf{x})$ if $\mathbf{x} \in \Omega$ and $\tilde{\eta}_{0}(\mathbf{x})=\eta_{1}$ if $\mathbf{x} \notin \Omega$. 
Notice that for this problem there is no longer any part of the boundary where the field $\tilde{\mathbf{u}}$ is outward. Therefore, a weak extended solution satisfies

$$
\begin{aligned}
\int_{0}^{\infty} \int_{\Omega \cup \Omega_{\text {out }}} & \eta\left(\frac{\partial \varphi}{\partial t}+\tilde{\mathbf{u}} \cdot \nabla \varphi\right) d t d x \\
= & \int_{\Omega \cup \Omega_{\text {out }}} \tilde{\eta}_{0}(\mathbf{x}) \varphi(0, \mathbf{x}) d x+\int_{0}^{\infty} \int_{\Gamma_{\text {in }}} \eta_{\text {in }}(t, \mathbf{x}) \varphi(t, \mathbf{x})\left|\mathbf{u}_{0} \cdot \mathbf{n}\right| d \sigma(x) d t
\end{aligned}
$$

for every $\varphi$ in $C_{0}^{\infty}\left(R^{D+1}\right)$.

Of course an extended solution of (4.1) is a weak solution.

THEOREM 4.1. Let $\mathbf{u}$ be a vector field in $\mathbf{V}$, and $\eta_{0}$ and $\eta_{\text {in }}$ be functions such that

$$
\begin{gathered}
\eta_{0} \in L^{\infty}(\Omega), \quad \eta_{\text {in }} \in L^{\infty}\left((0, T) \times \Gamma_{\text {in }}\right), \\
\eta_{0} \in\left\{\eta_{1}, \ldots, \eta_{n}\right\}, \quad \eta_{\text {in }} \in\left\{\eta_{1}, \ldots, \eta_{n}\right\} \text { a.e. }
\end{gathered}
$$

Then the problem (4.1) has a unique weak extended solution. Moreover, this weak extended solution is a renormalized solution and satisfies

$$
\begin{gathered}
\eta \in W^{1, \infty}\left(0, T ; L^{p}(\Omega)\right), \quad p<\infty, \\
\eta_{0} \in\left\{\eta_{1}, \ldots, \eta_{n}\right\} \text { a.e. }
\end{gathered}
$$

It has a trace $\eta_{\text {out }}$ on $\Gamma_{\text {out }}$ that belongs to $L^{\infty}\left(\Gamma_{\text {out }}\right)$, and

$$
\begin{aligned}
\int_{\Omega} \eta^{2}(t, \mathbf{x}) d x+\int_{0}^{t} \int_{\Gamma_{\text {out }}} \eta_{\text {out }}^{2}(s, \mathbf{x}) & \left|\mathbf{u}_{0} \cdot \mathbf{n}\right| d \sigma(x) d s \\
= & \int_{\Omega} \eta_{0}^{2}(\mathbf{x}) d x+\int_{0}^{t} \int_{\Gamma_{\mathrm{in}}} \eta_{\mathrm{in}}^{2}(s, \mathbf{x})\left|\mathbf{u}_{0} \cdot \mathbf{n}\right| d \sigma(x) d s .
\end{aligned}
$$

If $\mathbf{u}$ is smooth, a characteristics method is available for solving (4.1), and then (4.9) and (4.10) are easily obtained (see [2]).

Proof. We begin with the existence result. First we consider a regularized sequence $\mathbf{u}_{k}$ defined as follows. Since the restriction of $\tilde{\mathbf{u}}-\mathbf{U}_{0}$ to $\Omega$ belongs to $L^{\infty}(0, T ; \mathbf{V})$, there is a sequence $\mathbf{v}_{k}$ in $C_{0}^{\infty}(R \times \Omega)$ such that $\operatorname{div}\left(\mathbf{v}_{k}\right)=0$ and $\mathbf{v}_{k} \rightarrow \tilde{\mathbf{u}}-\mathbf{U}_{0}$ in $L^{p}\left(0, T ; H_{0}^{1}(\Omega)\right)$, with $p<\infty$. Then we define

$$
\begin{array}{ll}
\mathbf{u}_{k}=\mathbf{v}_{k}+\mathbf{U}_{0}, & \text { for } \mathbf{x} \in \Omega, \\
\mathbf{u}_{k}=\mathbf{U}_{0}\left(\theta_{\text {in }}+\theta_{\text {out }}\right), & \text { for } x \notin \Omega .
\end{array}
$$

It is easy to check that $\mathbf{u}_{k}$ belongs to $L^{\propto}\left(0, T ; C_{0}^{1}\left(R^{D}\right)\right)$. By a characteristics method we obtain a bounded sequence of solutions $\eta_{k}$ of (4.6) associated with the velocity field $\mathbf{u}_{k}$. Then it is easy to pass to the limit in (4.7) for a subsequence $\eta_{k}$ that converges in $L^{\infty}((0, T) \times \Omega)$ weak $*$. To prove uniqueness, let us consider a solution of (4.7) with $\eta_{0}=0, \eta_{\text {in }}=0$. We extend it by 0 outside $\Omega$ and obtain

$$
\int_{R^{D+1}} \eta\left(\frac{\partial \varphi}{\partial t}+\nabla \cdot(\tilde{\mathbf{u}} \varphi)\right) d t d x=0 .
$$

Then the result of [3, Theorem II.2] applies, which yields $\eta=0$. Now we prove that an extended solution of $(4.1)$ is a renormalized solution. Following [3], we introduce a 
regularized sequence

$$
\rho^{\varepsilon}(\mathbf{x})=\frac{1}{\varepsilon^{D}} \rho\left(\frac{\mathbf{x}}{\varepsilon}\right), \quad \rho \in C_{0}^{\infty}\left(R^{D}\right), \quad \int_{R^{D}} \rho d x=1,
$$

and we recall a result due to Friedrichs [5] (see also [3]).

Lemma 4.2. Let $\tilde{\mathbf{u}}$ in $L^{2}\left(0, T ;\left(H^{1}\left(R^{D}\right)\right)^{D}\right)$ and $\tilde{\eta}$ in $L^{\infty}\left((0, T) \times R^{D}\right)$. Then

$$
r^{\varepsilon}=\tilde{\mathbf{u}} \cdot \nabla\left(\tilde{\eta} * \rho^{\varepsilon}\right)-(\tilde{\mathbf{u}} \cdot \nabla \tilde{\eta}) * \rho^{\varepsilon} \rightarrow 0 \quad \text { in } L^{2}\left(0, T ; L_{\mathrm{loc}}^{2}\left(R^{D}\right)\right) .
$$

Let now $\eta$ be an extended solution of (4.1). The first step is to extend $\eta$ by a function $\tilde{\eta}$ of $L^{\infty}\left((0, T) \times R^{D}\right)$ that satisfies

$$
\frac{\partial \tilde{\eta}}{\partial t}+\tilde{\mathbf{u}} \cdot \nabla \tilde{\eta}=0, \quad t>0, \mathbf{x} \in R^{D}
$$

For that, we solve a "backward problem". Let $\omega=\Omega_{\text {in }}-\bar{\Omega}$. We solve

$$
\begin{aligned}
\frac{\partial \hat{\eta}}{\partial t}+\tilde{\mathbf{u}} \cdot \nabla \hat{\eta} & =0, & & t \in(0, T), \mathbf{x} \in \omega, \\
\hat{\eta}(T, \mathbf{x}) & =0, & & \mathbf{x} \in \omega, \\
\hat{\eta}(t, \mathbf{x}) & =\eta_{\text {in }}(t, \mathbf{x}), & & t \in(0, T), \mathbf{x} \in \Gamma_{\text {in }} .
\end{aligned}
$$

We point out that a solution of (4.13) is given by the preceding proof of existence and that

$$
\tilde{\mathbf{u}}=0, \quad \mathbf{x} \in \partial \omega-\Gamma_{\text {in }} .
$$

Therefore, for every function $\varphi$ in $C_{0}^{\infty}\left((0, T) \times R^{D}\right)$,

$$
\int_{0}^{T} \int_{\Omega} \hat{\eta}\left(\frac{\partial \varphi}{\partial t}+\nabla \cdot(\tilde{u} \varphi)\right) d t d x=-\int_{0}^{T} \int_{\Gamma_{\mathrm{in}}} \eta_{\mathrm{in}}(t, \mathbf{x}) \varphi(t, \mathbf{x})\left|\mathbf{u}_{0} \cdot \mathbf{n}\right| d \sigma(x) d t
$$

Then we define $\tilde{\eta}$ by

$$
\begin{aligned}
& \tilde{\eta}=\eta, \quad t \in(0, T), \mathbf{x} \in \Omega \cup \Omega_{\text {out }}, \\
& \tilde{\eta}=\hat{\eta}, \quad t \in(0, T), \quad \mathbf{x} \in \omega, \\
& \tilde{\eta}=0, \quad t \in(0, T), \quad \mathbf{x} \notin \Omega \cup \Omega_{\text {out }} \cup \Omega_{\text {in }} .
\end{aligned}
$$

It follows from (4.7) and (4.14) that $\tilde{\eta}$ satisfies (4.12). Define

$$
\eta^{\varepsilon}=\tilde{\eta} * \rho^{\varepsilon}
$$

We have

$$
\frac{\partial \eta^{\varepsilon}}{\partial t}+\tilde{\mathbf{u}} \cdot \nabla \eta^{\varepsilon}=r^{\varepsilon}, \quad t \in(0, T), \mathbf{x} \in R^{D}
$$

But $\eta^{\varepsilon}$ is smooth with respect to $t$ and $\mathbf{x}$, since $\frac{\partial \eta^{\varepsilon}}{\partial t}=-(\tilde{\mathbf{u}} \cdot \nabla \tilde{\eta}) * \rho^{\varepsilon}$. Therefore,

$$
\frac{\partial}{\partial t}\left(\beta\left(\eta^{\varepsilon}\right)\right)+\tilde{\mathbf{u}} \cdot \nabla \beta\left(\eta^{\varepsilon}\right)=r^{\varepsilon} \beta^{\prime}\left(\eta^{\varepsilon}\right), \quad t \in(0, T), \mathbf{x} \in R^{D},
$$

for every function $\beta$ in $C^{1}(R)$. Using Lemma 4.2 , we pass to the limit and obtain

$$
\frac{\partial}{\partial t}(\beta(\tilde{\eta}))+\tilde{\mathbf{u}} \cdot \nabla \beta(\tilde{\eta})=0 \quad \text { in } C_{0}^{\infty}\left((0, T) \times R^{D}\right) .
$$


It remains to study the traces of the sequence $\beta\left(\eta^{\varepsilon}\right)$ in order to determine the traces of $\beta(\eta)$. Choosing $\beta(t)=t^{2}$ in (4.15) we obtain

$$
\frac{\partial}{\partial t}\left(\left(\eta^{\varepsilon}-\eta^{\delta}\right)^{2}\right)+\tilde{\mathbf{u}} \cdot \nabla\left(\left(\eta^{\varepsilon}-\eta^{\delta}\right)^{2}\right)=2\left(r^{\varepsilon}-r^{\delta}\right)\left(\eta^{\varepsilon}-\eta^{\delta}\right)
$$

Let $\alpha$ be a nonnegative function in $C_{0}^{\infty}(R)$ such that

$$
\alpha=0 \quad \text { for } t \geq T, \quad \alpha=1 \quad \text { for } 0 \leq t \leq T^{*}<T .
$$

\section{Hence}

$$
\begin{gathered}
\int_{0}^{T} \int_{\Gamma_{\mathrm{in}}} \eta_{\mathrm{in}}\left(\eta^{\varepsilon}-\eta^{\delta}\right)^{2}\left|\mathbf{u}_{0} \cdot \mathbf{n}\right| \alpha(t) d \sigma(x) d t=\int_{0}^{T} \int_{\Omega \cup \Omega_{\mathrm{out}}}\left(\eta^{\varepsilon}-\eta^{\delta}\right)^{2}\left(\alpha^{\prime}(t)+\nabla \cdot \tilde{\mathbf{u}}\right) d x d t \\
+\int_{\Omega \cup \Omega_{\mathrm{out}}}\left(\tilde{\eta}_{0} * \rho^{\varepsilon}-\tilde{\eta}_{0} * \rho^{\delta}\right)^{2} d x+2 \int_{0}^{T} \int_{\Omega \cup \Omega_{\mathrm{out}}}\left(\eta^{\varepsilon}-\eta^{\delta}\right)\left(r^{\varepsilon}-r^{\delta}\right) \alpha(t) d x d t
\end{gathered}
$$

This proves that the trace of $\eta^{\varepsilon}$ on $\Gamma_{\text {in }}$ is a Cauchy sequence of the Hilbert space $L^{2}\left(0, T^{*} ; L^{2}\left(\Gamma_{\text {in }} ;\left|\mathbf{u}_{0} \cdot \mathbf{n}\right| d \sigma(x)\right)\right)$. Since this sequence is bounded, it converges in this space towards a bounded function $\tilde{\eta}_{\text {in }}$. The same integration on $\Omega_{\text {out }}$ proves that the trace of $\eta^{\varepsilon}$ converges in $L^{2}\left(0, T^{*} ; L^{2}\left(\Gamma_{\text {out }} ;\left|\mathbf{u}_{0} \cdot \mathbf{n}\right| d \sigma(x)\right)\right)$ towards a bounded function $\eta_{\text {out }}$. Then using the weak formulation

$$
\begin{aligned}
\int_{0}^{\infty} \int_{\Omega} \beta\left(\eta^{\varepsilon}\right)\left(\frac{\partial \varphi}{\partial t}+\nabla \cdot(\mathbf{U} \varphi)\right) d t d x \\
=\int_{0}^{\infty} \int_{\Omega} \beta^{\prime}\left(\eta^{\varepsilon}\right) r^{\varepsilon} \varphi d t d x+\int_{\Omega} \beta\left(\tilde{\eta}_{0} * \rho^{\varepsilon}\right)(\mathbf{x}) \varphi(0, \mathbf{x}) d t d x \\
\quad+\int_{0}^{\infty} \int_{\Gamma_{\text {in }}} \beta\left(\eta^{\varepsilon}\right) \varphi(t, \mathbf{x})\left|\mathbf{u}_{0} \cdot \mathbf{n}\right| d \sigma(x) d t \\
-\int_{0}^{\infty} \int_{\Gamma_{\text {out }}} \beta\left(\eta^{\varepsilon}\right) \varphi(t, \mathbf{x})\left|\mathbf{u}_{0} \cdot \mathbf{n}\right| d \sigma(x) d t
\end{aligned}
$$

we pass to the limit and obtain that $\beta(\eta)$ is a weak solution of $(4.1)$ with the data $\beta\left(\tilde{\eta}_{0}\right)$, $\beta\left(\tilde{\eta}_{\text {in }}\right)$. But taking $\beta(t)=t$ gives $\tilde{\eta}_{\text {in }}=\eta_{\text {in }}$. This proves that $\eta$ is a renormalized solution. Moreover, it has a bounded trace $\eta_{\text {out }}$ on $\Gamma_{\text {out }}$. Taking $\varphi=\alpha(t)$ on $\Omega$ in (4.16) and passing to the limit gives

$$
\begin{aligned}
\int_{0}^{\infty} \alpha^{\prime}(t) & \int_{\Omega} \beta(\eta)(t, \mathbf{x}) d t d x=\int_{\Omega} \beta\left(\eta_{0}\right)(\mathbf{x}) d x \\
\quad+ & \int_{0}^{\infty} \int_{\Gamma_{\text {in }}} \beta\left(\eta_{\text {in }}\right) \alpha(t)\left|\mathbf{u}_{0} \cdot \mathbf{n}\right| d \sigma(x) d t-\int_{0}^{\infty} \int_{\Gamma_{\text {out }}} \beta\left(\eta_{\text {out }}\right) \alpha(t)\left|\mathbf{u}_{0} \cdot \mathbf{n}\right| d \sigma(x) d t
\end{aligned}
$$

Choosing $\beta(t)=|t|^{p}$, it follows that $\eta(t, \cdot)_{L^{p}(\Omega)}$ belongs to $W^{1, \infty}(0, T)$. The above formula with $\beta(t)=t^{2}$ leads also to (4.10). To prove that $\eta$ takes its value in $\left\{\eta_{1}, \ldots, \eta_{n}\right\}$, we consider a function $\beta$, positive except for the value $\eta_{i}: \beta\left(\eta_{i}\right)=0, i=1, \ldots, N$. Then $\beta(\eta)$ is an extended solution of (4.1) with vanishing data. It follows from the result of uniqueness that $\beta(\eta)=0$, a.e. This concludes the proof of Theorem 4.1. 
5. Proof of the main theorem. Stokes problem (3.1) only provides some regularity on the space derivatives of $\mathbf{u}$, whereas the transport equation (4.1) gives some information on the time derivative of $\eta$. Therefore, some compensated compactness result seems to be appropriate for passing to the limit in the nonlinear term $\eta \mathbf{u}$.

LEMma 5.1. Let $\left(\eta^{n}\right)$ and $\left(\mathbf{v}^{n}\right)$ be two sequences uniformly bounded respectively in $L^{2}((0, T) \times \Omega)$ and $\left.L^{2}\left(0, T ; H_{0}^{1}(\Omega)\right)^{D}\right)$ such that

$$
\begin{array}{ll}
\eta^{n} \rightarrow \eta & \text { in } L^{2}((0, T) \times \Omega) \text { weak } \\
\mathbf{v}^{n} \rightarrow \mathbf{v} & \text { in } L^{2}\left(0, T ;\left(H_{0}^{1}(\Omega)\right)^{D}\right) \text { weak. }
\end{array}
$$

If $\frac{\partial \eta^{n}}{\partial t}$ is bounded in $L^{2}\left(0, T ; H^{-1}(\Omega)\right)$, then up to a subsequence, $\eta^{n} \mathbf{u}^{n}$ converges to $\eta \mathbf{u}$ in $\left(C_{0}^{\infty}((0, T) \times \Omega)\right)^{\prime}$.

Proof. $\left(\eta^{n}\right)$ and $\left(\frac{\partial \eta^{n}}{\partial t}\right)$ being bounded in $L^{2}\left(0, T ; L^{2}(\Omega)\right)$ and $L^{2}\left(0, T ; H^{-1}(\Omega)\right)$ respectively, Aubin's Lemma ([6]) implies that there is a subsequence of $\left(\eta^{n}\right)$ converging to $\eta$ in $L^{2}\left(0, T ; H^{-1}(\Omega)\right)$. We notice that the convergence of $\eta^{n}$ to $\eta$ in $L^{2}\left((0, T) ; H^{-1}(\Omega)\right)$ and the weak convergence of $\mathbf{v}^{n}$ to $\mathbf{v}$ in $L^{2}\left(0, T ;\left(H_{0}^{1}(\Omega)\right)^{D}\right)$ lead to the convergence of $\eta^{n} \mathbf{v}^{n}$ to $\eta \mathbf{u}$ in $\left(C_{0}^{\infty}((0, T) \times \Omega)\right)^{\prime}$.

Corollary 5.1. If $\mathbf{u}^{n}$ weakly converges to $\mathbf{u}$ in $L^{2}\left(0, T ;\left(H^{1}(\Omega)\right)^{D}\right)$ and $\left(\eta^{n}\right)$ is the solution of Theorem 4.1 associated with $\mathbf{u}^{n}$, then $\left(\eta^{n}\right)$ converges in $L^{2}((0, T) \times \Omega)$ to the solution of Theorem 4.1 associated with $\mathbf{u}$.

Proof. Introduce the norm

$$
\|\| \eta \|=\left[\|\eta\|_{L^{2}((0, T) \times \Omega)}+\int_{0}^{T}(T-t) \int_{\Gamma_{\text {out }}} \eta_{\text {out }}^{2}|\mathbf{u} \cdot \mathbf{n}| d \sigma(x) d t\right]^{1 / 2}
$$

and the space

$$
\left.\mathbf{X}=L^{2}((0, T) \times \Omega)\right) \cap L^{2}\left(0, T ; L^{2}\left(\Gamma_{\text {out }} ;\left|\mathbf{u}_{0} \cdot \mathbf{n}\right| d \sigma(x)\right)\right),
$$

provided with this norm \|\|$\|$. Thanks to $(4.10),\left(\eta^{n}\right)$ is bounded in $\mathbf{X}$. So, up to a subsequence, it weakly converges to some $\eta$ in $\mathbf{X}$. Lemma 5.1 applies to $\left(\eta^{n}\right)$ and $\left(\mathbf{u}^{n}-\mathbf{U}_{0}\right)$, since

$$
\frac{\partial \eta^{n}}{\partial t}=-\nabla \cdot\left(\eta^{n} \mathbf{u}^{n}\right)
$$

is bounded in $L^{2}\left(0, T ; H^{-1}(\Omega)\right)$. But $\eta^{n} \mathbf{U}_{0} \rightarrow \eta \mathbf{U}_{0}$ in $L^{\infty}$ weak star; therefore, $\eta^{n} \mathbf{u}^{n} \rightarrow$ $\eta \mathbf{u}$ in $\left(C_{0}^{\infty}((0, T) \times \Omega)\right)^{\prime}$. Then, passing to the limit in the formulation (4.7), we obtain that $\eta$ is the extended solution of (4.1) associated with the velocity field $\mathbf{u}$. It follows that the whole sequence weakly converges to $\eta$. We also conclude from (4.10) that

$$
\left\|\eta^{n}\right\|\|=\| \eta \eta \|
$$

Since the norm \|\|$\|$ is strictly convex, this leads to the strong convergence of $\eta^{n}$ to $\eta$. 
Let us now prove the main result:

Theorem 2.1 results from the existence of a fixed point for a map $\mathbf{F}$ by means of the Schauder theorem. This map is defined in the following way:

Let $C$ be the convex set of $L^{2}((0, T) \times \Omega)$ defined by

$$
C=\left\{\eta \in L^{\infty}((0, T) \times \Omega) \text { such that } 0<\eta_{1}<\eta(\mathbf{x}, t)<\eta_{N} \text { a.e. }\right\} .
$$

For every $\eta$ in $C$, let $\mathbf{u}$ be the unique solution of the Stokes problem associated to $\eta$, given by Theorem 3.1. Then $\mu=F(\eta)$ is defined as the unique extended solution given by Theorem 4.1, with an extended velocity field $\tilde{\mathbf{u}}$ defined as in (4.5). It follows from (4.9) that $F$ maps $C$ onto itself.

First we prove that $F$ is continuous: $\left(\eta^{n}\right)$ being a sequence of $C$ converging to $\eta$ in $L^{2}((0, T) \times \Omega)$, let $\mathbf{u}^{n}$ be the associated solution of $(3.1)$, and $\left(\mu^{n}\right)=\left(F\left(\eta^{n}\right)\right)$. From $(3.4)$, $\left(\mathbf{u}^{n}\right)$ is bounded in $L^{\infty}\left(0, T ;\left(H^{1}(\Omega)\right)^{D}\right)$; so there is a subsequence $\left(\mathbf{u}^{n_{p}}\right)$ and a $\mathbf{u}$ such that $\left(\mathbf{u}^{n_{p}}\right)$ weakly converges to $\mathbf{u}$ in $L^{2}\left(0, T ;\left(H^{1}(\Omega)\right)^{D}\right)$. Hence $\varepsilon\left(\mathbf{u}^{n_{p}}\right)$ weakly converges to $\varepsilon(\mathbf{u})$ in $\left(L^{2}((0, T) \times \Omega)\right)^{6}$. The strong convergence of $\left(\eta^{n_{p}}\right)$ to $\eta$ in $L^{2}((0, T) \times \Omega)$, as well as the weak convergence of $\varepsilon\left(\mathbf{u}^{n_{p}}\right)$ to $\varepsilon(\mathbf{u})$ in $L^{2}((0, T) \times \Omega)$ imply the convergence of $\eta^{n_{p}} \varepsilon\left(\mathbf{u}^{n_{p}}\right)$ to $\eta \varepsilon(\mathbf{u})$ in $L^{1}((0, T) \times \Omega)$. Therefore, passing to the limit in the Stokes problem (3.1), we obtain that $\mathbf{u}$ is the solution of the Stokes problem associated with $\eta$. Hence $\mathbf{u}$ is unique; so the whole sequence $\left(\mathbf{u}^{n}\right)$ weakly converges to $\mathbf{u}$ in $L^{2}\left(0, T ;\left(H^{1}(\Omega)\right)^{D}\right)$. On the other hand, $\left(F\left(\eta^{n}\right)\right)$ and $\left(\tilde{\mathbf{u}}^{n}\right)$ satisfy the assumptions of Corollary 5.1 , so that $\left(F\left(\eta^{n}\right)\right)$ converges in $L^{2}((0, T) \times \Omega)$ to the solution of Theorem 4.1 associated with $\mathbf{u}$, that is, exactly $F(\eta)$.

We now prove the compactness of $F$ : Let $\left(\eta^{n}\right)$ be an arbitrary sequence in $C$. Then the sequence of Stokes problem solutions $\left(\mathbf{u}^{n}\right)$ associated with $\left(\eta^{n}\right)$ is bounded in $L^{2}(0, T$; $\left.\left(H^{1}(\Omega)\right)^{D}\right)$. Hence there is a subsequence $\left(\mathbf{u}^{n_{p}}\right)$ weakly converging to some $\mathbf{U}$ in $L^{2}(0, T$; $\left.\left(H^{1}(\Omega)\right)^{D}\right)$. We conclude as above that, up to a subsequence, $F\left(\eta^{n}\right)$ strongly converges in $L^{2}((0, T) \times \Omega)$ towards the extended solution of $(4.1)$ associated with the velocity $\mathbf{u}$. It ends the proof of the compactness of $F$, as well as the proof of Theorem 2.1.

Acknowledgment. We would like to thank F. Murat for a helpful discussion and remarks.

\section{REFERENCES}

[1] G. Astarita and G. Marrucci, Principles of Non-Newtonian Fluid Mechanics, McGraw-Hill, New York, 1974

[2] C. Bardos, Problèmes aux limites pour les équations aux dérivées partielles du premier ordre à coefficients réels, Ann. Scient. Ecole Norm. Sup., 4ème série 3, 185-233 (1970)

[3] R. L. DiPerna and P. L. Lions, Ordinary differential equations, transport theory and Sobolev spaces, Invent. Math. 98, 511-547 (1989)

[4] A. Fortin, Y. Demay, and J. F. Agassant, Computation of stationary interfaces in coextrusion flows, Polymer Engrg. Sci. 34, 1101-1108 (1994)

[5] K. Friedrichs, Symmetric positive systems of differential equations, Comm. Pure Appl. Math. 7, 345-392 (1954)

[6] J. L. Lions, Quelques méthodes de résolution des problèmes aux limites non linéaires, GauthierVillars, 1969 
[7] O. A. Oleinik, Boundary value problems for elasticity theory in unbounded domains, Russian Math. Surveys 43 5, 65-119 (1988)

[8] R. Temam, Navier-Stokes Equations, North-Holland, Amsterdam, New York, Oxford, 1977

[9] S. F. Shen, Grapplings with the simulation of non-Newtonian flows in polymer processing, Internat. J. Numer. Methods Engrg. 34, 701-723 (1992)

[10] C. W. Hirt and B. D. Nichols, Volume of Fluids (VOF) method for the dynamics of free boundaries, J. Comput. Phys. 39, 201-225 (1981) 